\title{
Evolution of Individual Group Size Preference can Increase Group-level Selection and Cooperation
}

\author{
Simon T. Powers and Richard A. Watson
}

Natural Systems group, University of Southampton, U.K.; stp05r@ecs.soton.ac.uk

\begin{abstract}
The question of how cooperative groups can evolve and be maintained is fundamental to understanding the evolution of social behaviour in general, and the major transitions in particular. Here, we show how selection on an individual trait for group size preference can increase variance in fitness at the group-level, thereby leading to an increase in cooperation through stronger group selection. We are thus able to show conditions under which a population can evolve from an initial state with low cooperation and only weak group selection, to one where group selection is a highly effective force.
\end{abstract}

\section{Introduction}

Understanding how cooperative social groups can evolve and be maintained is a major challenge in both artificial life [1,2] and evolutionary biology [3, 4]. In particular, work on cooperative group formation has gained an impetus in recent years through a recognition of the major transitions in evolution [5], and the corresponding realisation that cooperation can be a driving force in evolution and not a mere curio [6]. The major transitions include the evolution of multi-cellular organisms from single cells, and the evolution of societies from solitary organisms. In these transitions, lower-level entities (single cells, solitary organisms) formed groups and donated part of their personal fitness to contribute to shared group success, to the point where cooperation amongst the lower-level entities was so great that the group ultimately became an individual in its own right $[5,6]$ (e.g., somatic cells give up independent reproductive rights in multicellular organisms, likewise for sterile workers in eusocial insect colonies). Such transitions therefore represent premier examples of cooperative group formation. From the viewpoint of artificial life, understanding the mechanisms behind these transitions, and cooperative group formation more generally, may help us to understand the evolution of increased complexity $[1,7]$, and to create applied systems where the evolution of a high degree of cooperation is supported [2].

The key difficulty that any explanation for the evolution of cooperative groups must overcome is this: if performing a group-beneficial cooperative act entails some cost, then why should an individual donate a component of its own fitness in order to contribute to the success of its group? Surely selfish cheats who do not themselves cooperate but instead simply reap the benefits of other group 
members' cooperation should be expected to be fitter, for they receive all of the group benefits whilst paying none of the individual costs. However cooperation can nevertheless evolve, even in the face of selfish cheats, if there is competition and hence selection between groups [8]. This is because groups with more cooperators will, all other things being equal, outcompete other groups that are dominated by selfish individuals. In this way, selection acting between groups provides an evolutionary force favouring cooperation. However, because selfish cheats still enjoy a relative fitness advantage within each group through freeriding, any between-group selection for cooperation is opposed by within-group selection for selfishness. In such a scenario selection is therefore multi-level, operating both within- and between- groups. The degree to which group cooperation evolves then depends on the extent to which selection between groups is stronger than selection within groups [8].

However, it is commonly held that the conditions under which between-group selection can overpower within-group selection are rather limited [9]. In particular, there must be a high variance in the proportion of cooperators between different groups, such that some groups contain many more cooperators, and are hence much more productive, than others. One way that this variance can be generated is if groups are formed assortatively, such that cooperators tend to form groups with other cooperators. This could happen, for example, if groups were founded by kin [10], for the genealogical relatives of a cooperator will themselves tend to be cooperators. Another way a high between-group variance can arise is if groups are founded by individuals sampled at random from the global population, but the number of founding individuals is small [11]. In this case the groups will start out as small, unrepresentative, samples of the population and so will tend to have a high variance. Selection at the group-level will be more effective as a result of this increased variance, and the increased effect of group selection would lead to greater cooperation. However, the initial group size must typically be very small if significant group-level variance and hence selection is to be generated by this mechanism.

We consider here how such groups with high variance, and that are consequently much affected by group selection, can evolve from an initial population where between-group variance is low and group selection weak. In order to do so we relax an assumption that is present in nearly all other multi-level selection models, namely, that initial group size must remain fixed over evolutionary time. Rather, we consider here the possibility that many organisms across all taxa may be able to influence the size of their group to some extent, through genetically coded (individual) traits. As an example, bacteria living in biofilms have a trait that controls the amount of extracellular polymeric substance that they secrete [12]. Since this substance allows bacterial cells to bind together, the amount produced could influence microcolony (group) size. In our model we then consider the evolution of both a group size preference trait such as this, and a behavioural trait that determines whether an individual cooperates or not, such that both traits evolve concurrently. If smaller groups lead, through increased group selection, to increased cooperation then individuals with a preference for 
founding smaller groups could potentially be selectively favoured, for they would experience a greater frequency of the benefits of cooperation in their groups and hence be on average fitter.

In our previous work [13] we showed that, in principle, individuals with a preference for a group size very much smaller than the current size could invade if they arose in a sufficiently high frequency. Such a sudden, large, decrease in group size is easier to evolve, since the increase in cooperation is greater if there is more of a decrease in group size. However, if group size decreased gradually, which seems a more plausible evolutionary mechanism, then it is not clear whether there could be sufficient immediate individual benefit to be selectively favoured. Here, we examine conditions under which groups of a smaller initial size can in fact evolve from larger ones, simply through gradual unbiased mutations on individual size preference, even when there are some other advantages to being in a larger group (e.g., better predator defence, access to resources that a smaller number of individuals cannot obtain [14]). We find that large jumps in size preference, as modelled by our previous work, are not required under (negative) frequency-dependant within-group selection. Consequently, this process can provide a gradualist explanation for the origin of cooperative groups.

\section{Modelling the Concurrent Evolution of Initial Group Size and Cooperative Behaviour}

The simulation model presented here considers a population structured as follows. Individuals reproduce within discrete groups for a number of generations, before individuals in all groups disperse and form a global migrant pool (dispersal stage). New groups are then formed by a uniform random sampling of individuals from this migrant pool, and the process of group growth, dispersal, and reformation repeats. Selection within groups occurs during the group growth stage, where individuals reproduce and have fitness-affecting interactions with other group members (i.e., selfish individuals will have more offspring than cooperators within that same group). On the other hand, selection between groups occurs at the dispersal stage, since those groups that have grown to a larger size (i.e., those with more cooperators) will contribute more individuals to the migrant pool. This kind of population structure is very similar to that considered in some classical group selection models $[15,11,10]$, and especially fits organisms that live on temporary resource patches which become depleted, thereby periodically forcing dispersal and the formation of new groups.

Unlike previous models, we give individuals a genetically coded initial group size preference. An individual genotype then contains two loci: the first codes for cooperative or selfish behaviour, the second for an initial group size preference. Mutation on these loci happens after the dispersal stage; double mutations within a single organism are not allowed. Mutation at the size locus is done by adding or subtracting 1 to the current size preference (with $50 \%$ probability each); mutation at the behaviour locus is done by swapping to the other behaviour. Groups are formed randomly with respect to behaviour (cooperative 
or selfish), but assortatively on size, such that individuals with a preference for smaller groups tend to, on average, find themselves in such groups, likewise for individuals with a preference for larger groups. This is implemented by creating a list of individuals in the migrant pool sorted ascendingly by their size preference. Individuals from this list are then added in order to a group, until the size of the group exceeds the mean preference of the group members, at which point a new group is created for the next individual and is populated in the same manner. An algorithmic overview of the model is as follows:

1. Initialisation: Let the migrant pool be the initial population.

2. Group formation: Assign individuals in the migrant pool to groups.

3. Reproduction: Perform reproduction and selection within groups for a fixed number of generations, using the fitness functions defined below. ${ }^{1}$

4. Dispersal: Place all individuals into the migrant pool.

5. Mutation: Mutate individuals in the migrant pool.

6. Iteration: Repeat from step 2 onwards until and equilibrium is reached.

\subsection{Within-group Selection: Snowdrift and Prisoner's Dilemma Games}

To model within-group selection and reproduction, we assume that group members have fitness-affecting interactions with each other as defined by the payoff matrix in Table 1 , where $b$ is the benefit of cooperating, and $c$ the cost. If $b>c$ then this represents the Snowdrift game [16], where a coexistence of cooperative and selfish individuals is supported at equilibrium in a freely-mixed population, since both types enjoy a fitness advantage when rare. Such an equilibrium coexistence of behaviours can occur if cooperators are able to internalise some of the benefits of cooperating. For example, if cooperation involves the production of a public good, then cooperators may be able to keep a fraction of the good they produce for themselves [17]. Where this occurs cooperators will receive, on average, a greater per capita share of the benefits of cooperation, giving them a fitness advantage. However, if the benefit of cooperation becomes discounted with additional cooperators, but the cost remains the same, then selfish individuals will become fitter as cooperators increase above a threshold frequency [17]. The Snowdrift game is thus a model of negative frequency-dependent selection leading to a mixed equilibrium; there is a growing realisation that this type of dynamic occurs in many biological systems $[16,17]$. It should be stressed that despite an equilibrium coexistence of behaviours, it is still the case that mean fitness would be higher if all individuals cooperated. This then means that if the game is played in a group structured population, groups with more cooperators will still be fitter than those with less. Group selection can, therefore, still further increase the global frequency of cooperation.

For $c>b>c / 2$, the payoff matrix in Table 1 yields the classical Prisoner's Dilemma [16], where selfish individuals are always fitter and cooperators are

\footnotetext{
${ }^{1}$ We rescale the groups after each generation to maintain a constant population size.
} 
Table 1. Payoff matrix for within-group interactions.

\begin{tabular}{|l|l|l|}
\hline & Cooperate & Selfish \\
\hline Payoff to Cooperate & $b-c / 2$ & $b-c$ \\
\hline Payoff to Selfish & $b$ & 0 \\
\hline
\end{tabular}

driven extinct at equilibrium in a freely-mixed population. Again, however, mean fitness would be higher if all individuals cooperated. We generalise the structure of this 2-player payoff matrix to a group of $n$ players by multiplying the payoff matrix by the proportion of behaviours within the group, as is standard when forming a replicator equation in evolutionary game theory; in our model this corresponds to treating each group as a separate freely-mixed population. Doing so yields the following fitness equations, where $w_{c}$ is the fitness of cooperators, $w_{s}$ the fitness of selfish individuals, $p$ the proportion of cooperators within the group, $w_{0}$ a baseline fitness in the absence of social interactions, and $\sigma_{n}$ a sigmoidal function that provides a benefit depending on group size $n$, as described below:

$$
\begin{aligned}
& w_{c}=p\left(b-\frac{c}{2}\right)+(1-p)(b-c)+w_{0}+\sigma_{n} \\
& w_{s}=p b+w_{0}+\sigma_{n} \\
& \sigma_{n}=\frac{\beta}{1+e^{-\mu n}}-\frac{\beta}{2}
\end{aligned}
$$

$\sigma_{n}$ is a sigmoidal function which takes in as input the current group size, and has as parameters a gradient $\mu$ (which determines how quickly the benefit tails off as the group gets larger), and a maximum fitness benefit $\beta$. This provides what is known in ecology as an Allee effect, whereby there is an advantage in number when groups are small, but as the group grows this is overwhelmed by the negative effects of crowding, i.e, the effect saturates with increasing size [14].

\section{Results}

We investigate here the conditions under which an individual preference for groups of a smaller initial size can evolve, and lead to greater group selection and cooperation. In all cases, we start all individuals out with the same size preference (20), and then consider the evolutionary dynamics that occur through mutation and selection. The initial frequency of the cooperation allele is taken to be the global equilibrium frequency in the model that occurs if group size was fixed at the starting size. We then record the changes in mean initial group size and global proportion of cooperators over time. A particular focus of this study is to contrast the effects of within-group selection modelled on the Snowdrift versus the Prisoner's Dilemma game. We set $b / c=1.1$ to yield the Snowdrift game, and $b / c=0.9$ to produce the Prisoner's Dilemma. The following parameter settings were used throughout: 3 generations within groups prior to dispersal, $w_{0}=1$, mutation rate $1 \%, 90 \%$ of mutations on the size locus, and population size 1000 . 
We initially considered a case where there is no intrinsic advantage to larger groups (i.e., set $\sigma_{n}=0$ ). We found that given time for a sufficient number of mutations to accumulate, a population could evolve down to an initial group size of 1 , and $100 \%$ cooperation, from a large range of initial conditions. The reason initial group size tended towards size 1 is that when there is no intrinsic benefit to larger groups, such an initial size maximises cooperation and hence absolute individual fitness. A representative example of the population dynamics is shown in Fig. 1. Interestingly, our results show that a selective gradient towards smaller groups does not always exist at the start; although it was present in the Snowdrift game, in the Prisoner's Dilemma the process relied on genetic drift until such a time as very small groups were created. This drift can be seen by individual size preferences spreading out in both directions at the start, whereas in the Snowdrift game the mass of the population moved in one direction, thus showing the presence of an individual selective gradient favouring smaller groups from the outset.
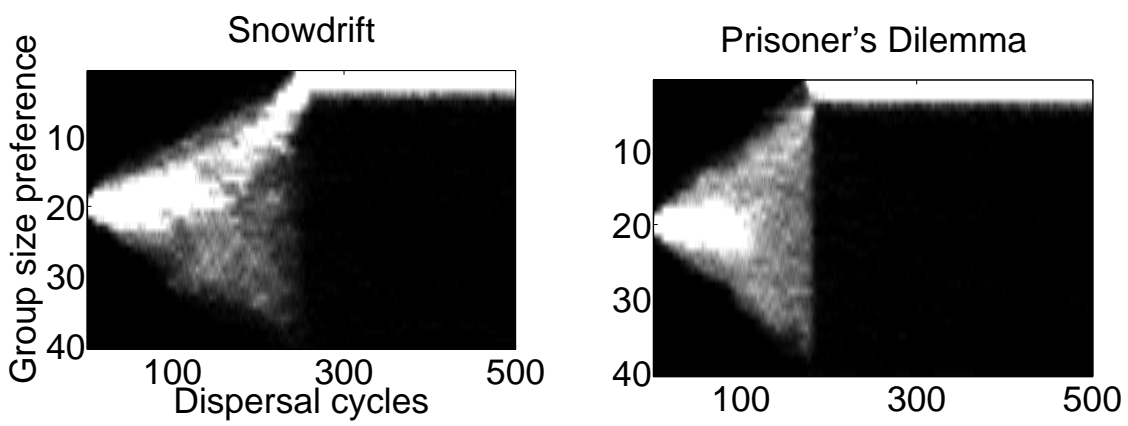

Fig. 1. Change in group size preference under Snowdrift and Prisoner's Dilemma games (white $=$ majority of population).

Next, we considered the case where there is an intrinsic advantage to larger groups $\left(\sigma_{n}\right.$ parameters: $\beta=1$ and $\left.\mu=0.4\right)$. In such cases, the optimum initial group size (in terms of absolute individual fitness) is a trade-off between the benefit of cooperation and the intrinsic advantage to larger groups. It will thus be greater than 1, but not so large as to prevent any significant between-group selection and hence cooperation: using the parameters here, the optimum is 4 . The question is then whether this optimum size can be reached by mutation. Figure 2 shows an example of initial conditions under which mean group size can decrease to the optimum in the Snowdrift, but not Prisoner's Dilemma, game. The reason size preference cannot decrease in the Prisoner's Dilemma is that drift can no longer be effective if there is an intrinsic advantage to larger groups, for the advantage of larger groups provides a selective pressure away from small. By contrast, in the Snowdrift game a counter selective gradient towards smaller groups and increased cooperation exists from a much larger 
range of initial conditions. This counter gradient is provided by the benefits of increased cooperation that can be realised in smaller groups due to increased group selection. In the Prisoner's Dilemma there is no such gradient because group selection is completely ineffective over much of the parameter space, and so a small mutation on size would not lead to any increase in cooperation.
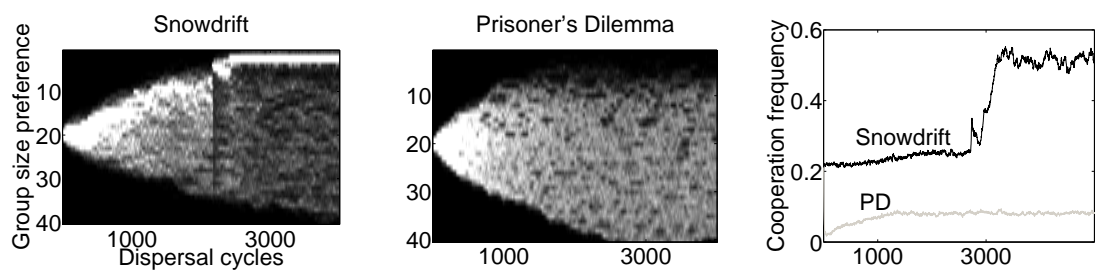

Fig. 2. Allowing for some benefit to larger groups. Left and centre: change in group size preference (white $=$ majority of population). Right: proportion of cooperators.

The existence of a selective gradient over a larger range of parameters follows from the fact that the Snowdrift game maintains a low frequency of cooperators at (group) equilibrium. Consequently, because one type cannot be driven extinct there is always the possibility that group-level variance can be generated when the groups are reformed, even when groups are large, as shown in some of our previous work [18]. As a result, moving to a slightly smaller group size can further increase this variance, and allow increased group selection and a subsequent increase in cooperation. By contrast, in the Prisoner's Dilemma game moving to a slightly smaller group size would not increase the effect of group selection over much of the parameter space, for cooperators are driven extinct over a large range of group sizes, thereby destroying the possibility of any group variance. Where this occurs, there cannot be selection for a smaller group size, and so the process must rely on drift, which cannot overcome an intrinsic advantage to larger groups. Our results therefore demonstrated that the evolution of smaller initial group size and greater cooperation is much more plausible if within-group selection takes the form of a Snowdrift game.

\section{Concluding Remarks}

Many of the major transitions involved the evolution of mechanisms that increased variance and hence selection at the group level, thereby allowing a high degree of cooperation between group members to evolve [6]. One such mechanism could be a reduction in initial group size. For example, multi-cellular organisms are themselves cooperative groups of individual cells. Most multi-cellular organisms develop from a single cell, and it has been argued that this evolved at least partly because it increased between-organism (cell group) variance, and hence increased cooperation between individual cells within the organism $[5,6]$. We 
have shown here how selection on an individual trait can lead to the evolution of increased variance in fitness at the group level, and hence a rise in cooperation between group members through increased group selection. Our results demonstrated that such a process is much more plausible if negative frequencydependent selection, as modelled here by the Snowdrift game, operates within groups.

Acknowledgements Thanks to Alex Penn and Seth Bullock for many discussions, and Rob Mills for feedback on the manuscript.

\section{References}

1. Stewart, J.: Evolutionary transitions and artificial life. Artificial Life 3(2) (1997) $101-120$

2. Nitschke, G.: Emergence of cooperation: State of the art. Artificial Life 11 (2005) 367-396

3. Hammerstein, P.: Genetic and Cultural Evolution of Cooperation. MIT Press (2003)

4. West, S.A., Griffin, A.S., Gardner, A.: Evolutionary explanations for cooperation. Current Biology 17(16) (2007) R661-R672

5. Maynard Smith, J., Szathmáry, E.: Major Transitions in Evolution. Spektrum (1995)

6. Michod, R.E.: Darwinian Dynamics: Evolutionary Transitions in Fitness and Individuality. Princeton University Press (1999)

7. Bedau, M.A.: Artificial life: organization, adaptation, and complexity from the bottom up. Trends in Cognitive Science 7 (2003) 505-512

8. Wilson, D.S., Sober, E.: Reintroducing group selection to the human behavioral sciences. Behavioral and Brain Sciences 17(4) (1994) 585-654

9. Maynard Smith, J.: Group selection. Quarterly Review of Biology 51 (1976) $277-283$

10. Wilson, D.S.: Altruism in mendelian populations derived from sibling groups: The Haystack model revisited. Evolution 41(5) (1987) 1059-1070

11. Wilson, D.S., Colwell, R.K.: Evolution of sex ratio in structured demes. Evolution 35(5) (1981) 882-897

12. Flemming, H.C., Neu, T.R., Wozniak, D.J.: The EPS matrix: The House of Biofilm Cells. Journal of Bacteriology 189(22) (2007) 79457947

13. Powers, S.T., Penn, A.S., Watson, R.A.: Individual selection for cooperative group formation. In: Proceedings of ECAL 2007, Springer (2007) 585-594

14. Avilés, L.: Cooperation and non-linear dynamics: An ecological perspective on the evolution of sociality. Evolutionary Ecology Research 1 (1999) 459-477

15. Maynard Smith, J.: Group selection and kin selection. Nature 201 (1964) 11451147

16. Doebeli, M., Hauert, C.: Models of cooperation based on the prisoner's dilemma and the snowdrift game. Ecology Letters 8(7) (2005) 748-766

17. Gore, J., Youk, H., van Oudenaarden, A.: Snowdrift game dynamics and facultative cheating in yeast. Nature 459 (2009) 253-256

18. Powers, S.T., Penn, A.S., Watson, R.A.: The efficacy of group selection is increased by coexistence dynamics within groups. In: Proceedings of ALife XI, MIT Press (2008) 498-505 